\title{
Note on the method of finding the particular integral of the differential Equation
}

$$
f(\mathrm{D}) y={\stackrel{n}{N} a_{r} x^{x}}^{*}
$$

By D. K. Picken.

The use of inverse operators is only justifiable when it is obvious what direct operation of the calculus they symbolise.

The purpose of this note is to point out how the usual method of obtaining the integral of this differential equation can be shown as the result of direct operations.

We suppose that

$$
\begin{aligned}
f(z) & =\mathbf{A}_{0}+\mathbf{A}_{1} z+\ldots+\mathbf{A}_{m} z^{m}, \text { where } \mathbf{A}_{0}, \mathbf{A}_{1} \ldots \mathbf{A}_{m} \text { are constants, } \\
& =\frac{1}{\phi(z)} .
\end{aligned}
$$

Let $\psi(z)=\phi(0)+z \phi^{\prime}(0)+\ldots+\frac{z^{n}}{n !} \phi^{(n)}(0)$, a polynomial of degree $n$; then it is easy to show by Leibnitz's Theorem, if it is not otherwise obvious, that

$$
f(z) \cdot \psi(z)=1+z^{n+1} \cdot \mathrm{R}_{m-1}(z) \text {, where } \mathrm{R}_{m-1}(z)
$$

is a polynomial of degree $(m-1)$;

$$
\begin{aligned}
\therefore f(\mathrm{D}) y & =\sum_{1}^{n} a_{r} x^{r} \\
& =[f(\mathrm{D}) \cdot \psi(\mathrm{D})] \sum_{1}^{n} a_{r} x^{r} ;
\end{aligned}
$$

i.e. $y=\psi(\mathrm{D}) . \sum_{1}^{n} a_{1} x^{r}$, which is the usual rule. 